\title{
X-ray emission from expanding cocoons
}

\author{
C. Zanni ${ }^{1,2}$, G. Bodo $^{2}$, P. Rossi ${ }^{2}$, S. Massaglia ${ }^{1}$, A. Durbala ${ }^{1,2,3}$, and A. Ferrari ${ }^{1}$ \\ 1 Dipartimento di Fisica Generale dell'Università, Via Pietro Giuria 1, 10125 Torino, Italy \\ 2 INAF - Osservatorio Astronomico di Torino, Strada dell'Osservatorio 20, 10025 Pino Torinese, Italy \\ 3 Universitatea din Bucuresti, Facultatea de fizica atomica si nucleara, Bucuresti-Magurele, \\ PO Box MG-11, Romania, Italy
}

Received 3 December 2002 / Accepted 21 February 2003

\begin{abstract}
X-ray observations of extragalactic radiosources show strong evidences of interaction between the radio emitting plasma and the X-ray emitting ambient gas. In this paper we perform a detailed study of this interaction by numerical simulations. We study the propagation of an axisymmetric supersonic jet in an isothermal King atmosphere and we analyze the evolution of the resulting X-ray properties and their dependence on the jet physical parameters. We show the existence of two distinct and observationally subsequent different regimes of interaction, with strong and weak shocks. In the first case shells of enhanced X-ray emission are to be expected, while in the second case we expect deficit of X-ray emission coincident with the cocoon. By a comparison between analytical models and the results of our numerical simulations, we discuss the dependence of the transition between these two regimes on the jet parameters and we find that the mean controlling quantity results to be the jet kinetic power. We then discuss how the observed jets can be used to constrain the jet properties.
\end{abstract}

Key words. X-rays: galaxies: clusters - galaxies: jets - hydrodynamics

\section{Introduction}

X-ray observations of extragalactic radio sources have revealed strong evidences of interaction between the radio emitting plasma and the X-ray emitting gas in the ambient medium. The observation of Cygnus A with the ROSAT HRI (High Resolution Imager) by Carilli et al. (1994) and with the Chandra X-ray Observatory (Smith et al. 2002) showed deficits of X-ray emission in the cluster gas spatially coincident with the radio lobes. Observations of the Perseus cluster by Böhringer et al. (1993), also with ROSAT, and by Fabian et al. (2000) with the Chandra X-ray Observatory showed that the cluster emitting gas was displaced by the radio lobes of the source NGC 1275. A similar behavior has been observed in the Hydra A cluster, hosting the radio source 3C 218, with the Chandra X-ray Observatory by McNamara et al. (2000) (see also Nulsen et al. 2002), and in Abell 2052 that shows regions devoid of $\mathrm{X}$-ray emission coincident with the radio lobes of 3C 217 (Blanton et al. 2001). Other clear examples of the interaction between radio lobes and the surrounding cluster gas are given by A 4059 (Heinz et al. 2002, Chandra) and A 2199 (Owen \& Eilek 1998, ROSAT). Disturbances by a radio source are also found in the gas halo of some giant elliptical galaxies such as M 87 (Böhringer et al. 1995, ROSAT) and M 84 (Finoguenov \& Jones 2001, Chandra).

Send offprint requests to: G. Bodo, e-mail: bodo@to. astro.it
On the other hand, theoretical models predict that jets in radiogalaxies inflate overpressured cocoons that displace and compress the ambient gas and the effects of such interaction could indeed expected to be the formation of cavities and shells in the X-ray emission, as shown by observations. A simple onedimensional model of this interaction has been presented by Begelman \& Cioffi (1989). More detailed and realistic models require the use of numerical simulations. In this context, Clarke et al. (1997) carried out calculations of the jet propagation in a King atmosphere obtaining simulated X-ray images to compare with ROSAT data on Cygnus A. They demonstrated that a deficit in the X-ray brightness is indeed shown in the simulation results and found agreement between simulations and observations for moderate Mach number of the jet $(M \gtrsim 4)$. A similar scenario is depicted by the numerical simulations of Rizza et al. (2000) that showed the interaction and disruption of a jet inside a cooling flow cluster. More recently Reynolds et al. (2001) have pointed out that cocoons start being strongly overpressured, but, during their evolution, their pressure decreases, and they then become essentially in pressure equilibrium with the ambient or even underpressured. During this evolution, therefore, the shock driven in the external medium is strong at the beginning and becomes very weak at the end. Reynolds et al. (2001) call this last phase "sonic boom". The need for weak shocks comes from the observations of cool rims surrounding some of the X-ray cavities (see A 2052, Hydra A, Perseus A) that rule out the possibility of strong shocks driven 
by the expanding cocoon. To explain these observations several analytical (Churazov et al. 2000; Soker et al. 2002) and numerical (Churazov et al. 2001; Brighenti \& Mathews 2002; Quilis et al. 2001) models of "bubbles" of hot plasma expanding subsonically in the ambient medium have been studied. If these bubbles are buoyant they can also explain the presence of deficits of X-ray emission far from the radio lobes as observed in Perseus A. On the other hand, Heinz et al. (1998) and Alexander (2002) have studied self-similar solutions of simplified analytical models of overpressured cocoons expanding in a stratified medium in order to explain the observed features.

In this paper we analyze in detail, by using numerical simulations, the evolution of the X-ray properties of expanding cocoons and their dependence on the jet properties. The jets are characterized by their Mach number and their density ratio with the ambient medium density; the parameter plane is widely covered in order to consider a wide range of jet powers. We confirm the results presented by Reynolds et al. (2001) on the existence of two distinct and observationally different subsequent regimes of interaction, with strong or weak shocks but we are able to determine how and when the transition between these two regimes occurs, depending on the jet parameters. These results, on the other hand, show how the X-ray properties of cocoons could possibly be used as diagnostic for the jet characteristics.

The plan of the paper is the following: in Sect. 2 we describe the model, the basic equations and the initial conditions, in Sect. 3 we discuss the X-ray morphologies resulting from the simulations, in Sect. 4 we discuss the heating of the external material compressed by the expanding cocoon and the consequent changes in the X-ray emission properties, in Sect. 5 we discuss the physics of the cocoon expansion that leads to the interpretation of the different X-ray morphologies and in Sect. 6 we discuss the astrophysical relevance of our results, finally a summary is presented in Sect. 7.

\section{Numerical simulations}

We solve numerically the hydrodynamic equations for a supersonic jet, in cylindrical (axial) symmetry in the coordinates $(r, z)$ continuously injected into a gravitationally stratified (but not self-gravitating) medium

$$
\begin{aligned}
& \frac{\partial \rho}{\partial t}+\nabla \cdot(\rho \boldsymbol{v})=0 \\
& \frac{\partial \boldsymbol{v}}{\partial t}+(\boldsymbol{v} \cdot \nabla) \boldsymbol{v}=-\nabla p / \rho+\nabla \phi \\
& \frac{\partial p}{\partial t}+(\boldsymbol{v} \cdot \nabla) p-\gamma \frac{p}{\rho}\left[\frac{\partial p}{\partial t}+(\boldsymbol{v} \cdot \nabla) \rho\right]=0
\end{aligned}
$$

where the fluid variables $p, \rho, \boldsymbol{v}$ and $E$ are, as customary, pressure, density, velocity, and thermal energy $(p /(\gamma-1))$ respectively; $\gamma$ is the ratio of the specific heats. Radiative losses are neglected since the estimated radiative times are much longer than the evolution time scale.

The system of Eqs. (1) has been solved numerically employing a PPM (Piecewise Parabolic Method) hydrocode (Woodward \& Colella 1984). The integration domain has a size $r_{\text {domain }} \times z_{\text {domain }}$ where $r_{\text {domain }}=z_{\text {domain }}=2.6 a$, where $a$ is the core radius, defined below, and has been divided in $1024 \times 1024$ grid points. The axis of the jet is along the left boundary of the domain $(r=0)$, where we have imposed symmetric boundary conditions for $p, \rho, v_{z}$ and antisymmetric conditions for $v_{r}$. Reflective boundary conditions are also imposed on the boundary of injection of the jet $(z=0)$ outside its radius in order to reproduce a bipolar flow and to avoid spurious inflow effects. Free outflow is set on the remaining boundaries by imposing a null gradient for each variable $(\mathrm{d} / \mathrm{d} r=0)$.

The undisturbed ambient medium is assumed stratified in a spherically symmetric gravitational well, according to a classical isothermal King model:

$\rho_{\mathrm{ext}}(R)=\frac{\rho(0)}{\left[1+(R / a)^{2}\right]^{3 \beta / 2}}$,

with $R=\sqrt{r^{2}+z^{2}}$ and $\beta=0.5$. The resulting pressure stratification is kept in equilibrium by an appropriated external gravitational potential.

A (cylindrical) jet is injected from the bottom boundary of the integration domain, in pressure balance with the ambient. The initial jet velocity profile has the form

$v_{z}(r)=\frac{v_{\mathrm{j}}}{\cosh \left[(20 r / a)^{m}\right]}$

and the corresponding density profile is

$\rho(r, z)=\frac{\rho_{\mathrm{j}}-\rho_{\mathrm{ext}}(R)}{\cosh \left[(w 20 r / a)^{n}\right]}+\rho_{\mathrm{ext}}(R)$

with $w=0.77, m=8$ and $n=2 m$, giving a jet radius $r_{\mathrm{j}}=a / 20$.

Measuring lengths in units of the core radius $a$, velocities in units of the adiabatic sound speed $c_{\mathrm{se}}$ in the undisturbed external medium and the density in units of the ambient central density $\rho(0)$, our main parameters are the Mach number $M \equiv v_{\mathrm{j}} / c_{\mathrm{se}}$ and the density ratio $v=\rho_{\mathrm{j}} / \rho(0)$. Consistently the unit for the kinetic power is

$$
\begin{aligned}
& L_{\mathrm{k}}=\frac{\pi}{2} \rho(0) r_{\mathrm{j}}^{2} c_{\mathrm{se}}^{3} \\
& =1.5 \times 10^{42}\left(\frac{n_{0}}{10^{-2} \mathrm{~cm}^{-3}}\right)\left(\frac{a}{50 \mathrm{kpc}}\right)^{2}\left(\frac{T}{3 \mathrm{keV}}\right)^{3 / 2} \mathrm{erg} \mathrm{s}^{-1}
\end{aligned}
$$

and the unit of time is

$t_{0}=\frac{a}{c_{\mathrm{se}}}=4.8 \times 10^{7}\left(\frac{a}{50 \mathrm{kpc}}\right)\left(\frac{T}{3 \mathrm{keV}}\right)^{-1 / 2}$ years

where $n_{0}$ is the central electron density, and we will measure time $\tau$ in units of $t_{0}$. Then the kinetic power of the jet expressed in unity of $L_{\mathrm{k}}$ is given by

$L_{\mathrm{j}}=M^{3} v L_{\mathrm{k}}$

In Table 1 we show the parameters $M$ and $v$ for the simulations that we have performed giving also the corresponding values of the kinetic jet power $L_{\mathrm{j}}$ in units of $L_{\mathrm{k}}$ and the final simulation times in units of $t_{0}$. 
Table 1. Parameters of the seven simulations performed: Mach number $M$, density ratio $v=\rho_{\mathrm{j}} / \rho(0)$, kinetic jet power $L_{\mathrm{j}} / L_{\mathrm{k}}=M^{3} v$ and final time of the simulation $\tau_{\text {fin }}=t_{\text {fin }} / t_{0}$.

\begin{tabular}{cccc}
\hline \hline$M$ & $v$ & $L_{j} / L_{k}$ & $\tau_{\text {fin }}$ \\
\hline 10 & 0.1 & $10^{2}$ & 1.38 \\
60 & 0.001 & $2.16 \times 10^{2}$ & 1.22 \\
60 & 0.01 & $2.16 \times 10^{3}$ & 0.49 \\
60 & 0.1 & $2.16 \times 10^{4}$ & 0.15 \\
120 & 0.001 & $1.73 \times 10^{3}$ & 0.76 \\
120 & 0.01 & $1.73 \times 10^{4}$ & 0.25 \\
120 & 0.1 & $1.73 \times 10^{5}$ & 0.07 \\
\hline
\end{tabular}

\section{X-ray signatures: Shell and cavity}

The general structure of the interaction between a low density jet and the ambient medium is well known since the first simulations of Norman et al. (1982) (see also Massaglia et al. 1996 and Krause 2003). The flowing jet matter, slowed down by one or more terminal shocks, inflates a cocoon that compresses and drives shocks in the surrounding external medium. The compressed ambient material forms a shell surrounding the cocoon: the boundary between the shell and the cocoon is marked by a contact discontinuity, while the boundary between the shell and the undisturbed external medium is marked by a shock. We will call "extended cocoon" the whole region interested in the interaction between the jet and the ambient medium. The "extended cocoon" is then formed by the cocoon proper and by the surrounding shell. The cocoon, which is formed by the expanded jet material forms a cavity with very low density and high temperature, in which the X-ray emissivity is strongly depressed. On the other hand the external material in the shell has an enhanced emission due to its compression. In addition, depending on the external shock strength this material can be heated and its emission properties can then change.

In principle, we then expect three main features in the X-ray properties of the region of interaction between a jet and the ambient medium:

1. a region of depressed emission coincident with the cocoon;

2. a shell of enhanced emission;

3. a variation in the spectral properties of the emission by the shell.

The actual appearance, however, will depend on an interplay between all these effects. In fact, we have to take into account that what we see is an integration along the line of sight, that can go both through the cocoon and through the shell. Therefore, it can happen that the enhanced shell emission can compensate the deficit of emission in the cocoon and, in these cases, in correspondence of the cocoon we can even see a flux higher than that expected in the undisturbed case.

In Figs. 1-3 we show in the first two columns simulated X-ray flux distribution for the seven cases described in Table 1. The figures are symmetrical with respect to the $r$ and $z$ axes. The emissivity per frequency unit is computed with a Raymond-Smith thermal spectrum code (see HEASARC
Web Page) from the electron density and temperature distribution obtained in our calculation. The emissivity $\epsilon=n^{2} \Lambda(T)$ is then integrated in the $0.1-4 \mathrm{keV}$ band. The flux distribution is calculated integrating the optically thin emissivity along the line of sight $\lambda$ that is assumed perpendicular to the jet axis:

$f=\int_{-r_{\text {domain }}}^{+r_{\text {domain }}} \epsilon(n, T, \lambda) \mathrm{d} \lambda$.

We consider an isothermal ambient medium with a temperature $T=2.3 \mathrm{keV}$ and a central electron density $n_{0}=0.01 \mathrm{~cm}^{-3}$. In Fig. 1 we show the results for the three cases with $M=120$, each row refers to a different value of the density ratio and more precisely the top row is for $v=0.1$, the middle row is for $v=0.01$ and the bottom row is for $v=0.001$. The first two panels in a row are for a different time and we have chosen the times in order to have lengths respectively of 1 and 2 core radii. Figure 2 has the same structure, but is for the cases with $M=60$, while Fig. 3 is for the single case with $M=10$ and $v=0.1$. A more quantitative view of these results can be obtained presenting cuts of these figures along selected directions. In particular in Figs. 1-3 in the third and fourth column we show longitudinal cuts of the images along the jet axis. The figures correspond to the images of the first two columns and refer respectively to $M=120, M=60$ and $M=10$. The dashed curves in all the figures represent the emission from the undisturbed atmosphere.

These figures show a sequence of morphologies starting from cases in which a cavity is not present going to cases in which the cavity is the dominant feature of the image. In particular, we see that for $M=120$ the brightness depression is evident only for the lighter case, while for the $M=60$ cases starts to be present for $v=0.01$ during its evolution and is very evident during the whole evolution for $v=0.001$. For $M=10$ the cavity is dominant already for $v=0.1$.

The presence or absence of the brightness depression, as discussed by Clarke et al. (1997) depends on the thickness of the shell. In fact, the line of sight that goes through the cocoon region with very low emissivity crosses also the shell of enhanced emissivity, and the observed flux is higher or lower than the undisturbed profile depending on the interplay between the two effects. If the shell is narrow, as a consequence of mass conservation, it will have a high density, the emissivity will be greatly enhanced and will overcome the decrease in the cocoon giving an observed brightness higher than the undisturbed profile. Following Clarke et al. (1997), we can write the ratio of the observed flux to the undisturbed one as

$\frac{f^{\prime}}{f}=\frac{1}{\delta(2-\delta)^{2}}$

where we have neglected the dependence of emissivity on the temperature and $\delta$ is the ratio between the shell width and the cocoon radius. From Eq. (8), we see that the ratio increases as $\delta$ goes to 0 and becomes less than 1 for $\delta>0.38$.

In Fig. 4 we show the density distribution for the $M=$ 60 cases: each row refers to a different density ratio $(v=$ $0.1,0.01,0.001$ from top to bottom) and each column is for different times corresponding to cocoon lengths $1,1.5,2$ respectively. We see in fact that the width of the shell increases going 

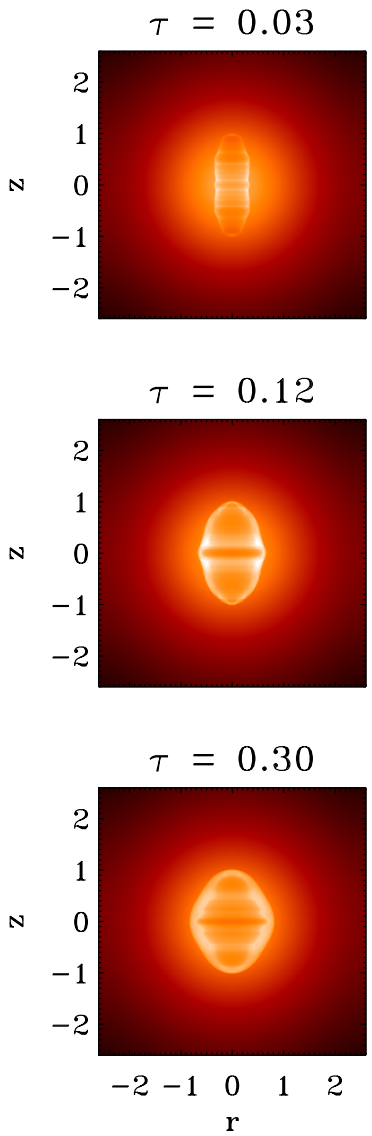

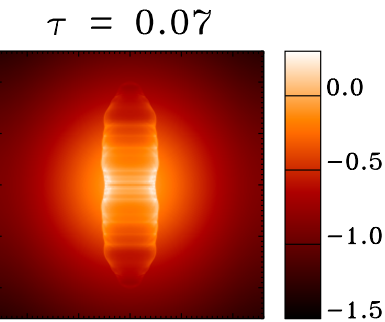

$\tau=0.22$

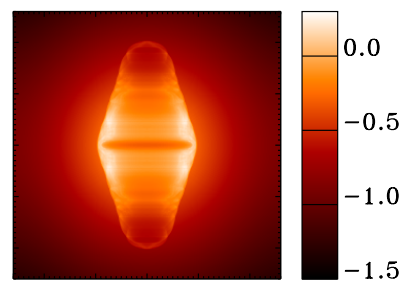

$\tau=0.69$

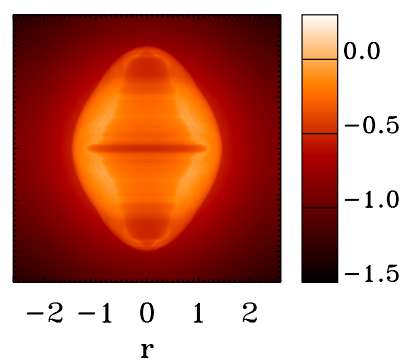

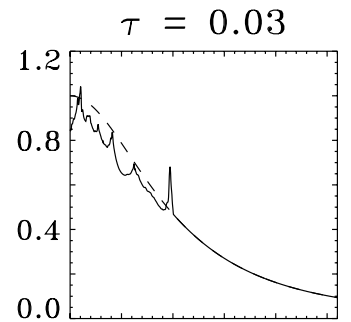
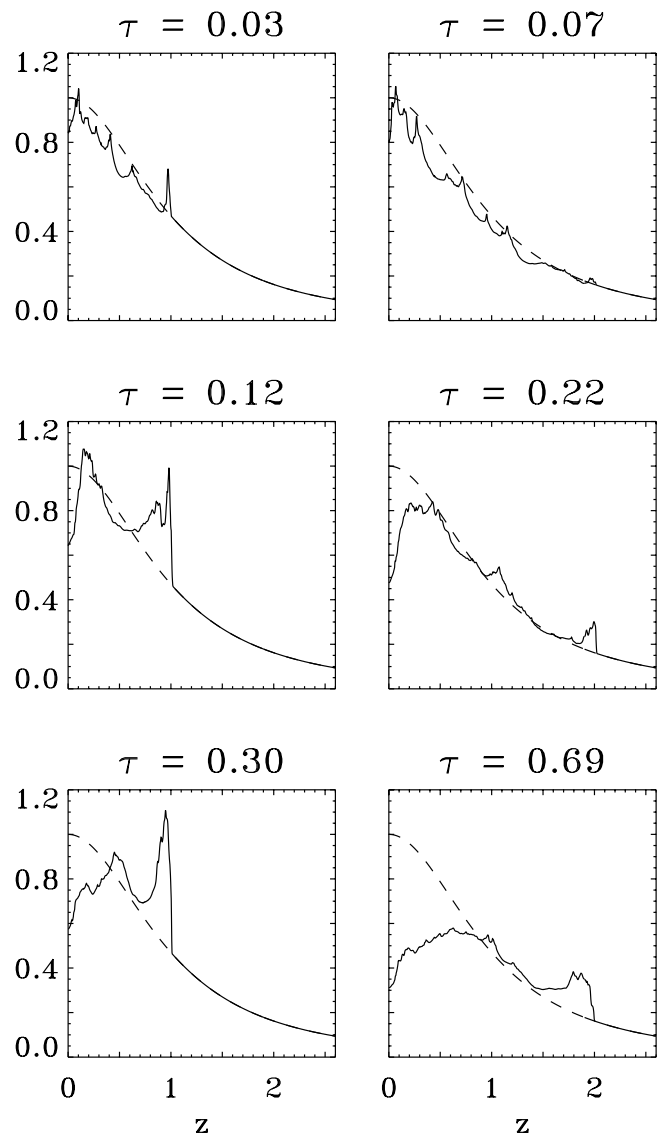

Fig. 1. Simulated X-ray fluxes in the $0.1-4 \mathrm{keV}$ band for the $M=120$ cases. The rows refer from top to bottom to the $v=0.1,0.01,0.001$ cases respectively. In the first two columns on the left the X-ray images are shown in logarithmic scale at times corresponding to cocoon lengths of $l_{\mathrm{c}} \sim 1$ and 2 core radii. In the two columns on the right longitudinal cuts along to the jet axis corresponding to the images on the left are plotted with a solid line. The dashed line represents the emission from the undisturbed atmosphere. The fluxes are given in unity of the central flux of the unperturbed atmosphere.

from the high to the low $v$ case. Moreover we can see that the shell tends to widen and the bow shock becomes weaker during the evolution of the cocoon, apart from the $v=0.1$ case in which the relative thickness of the shell remains constant. Note that the localized feature appearing along the $z=0$ axis is due to the assumed reflection boundary conditions and does not affect the general morphological and dynamical behavior.

In order to better quantify the deficit or the enhancement in the X-ray emission, we have computed integral measures $K_{\mathrm{c}}$ and $K_{\mathrm{s}}$ of these quantities defined as

$$
\begin{aligned}
& K_{\mathrm{c}}=\frac{1}{S_{\mathrm{c}}} \int_{S_{\mathrm{c}}}\left(f-f_{\mathrm{k}}\right) \mathrm{d} S \\
& K_{\mathrm{s}}=\frac{1}{S_{\mathrm{s}}} \int_{S_{\mathrm{s}}}\left(f-f_{\mathrm{k}}\right) \mathrm{d} S,
\end{aligned}
$$

where the surface integrals are computed over the domains $S_{\mathrm{c}}$ and $S_{\mathrm{s}}$ defined as the areas over which the integrand $\left(f-f_{\mathrm{k}}\right)$ is respectively lower (the cavity) and greater (the shell) than zero, where $f$ is the flux at a given position and $f_{\mathrm{k}}$ is the flux at the same position for an unperturbed King atmosphere. The quantities $K_{\mathrm{c}}$ and $K_{\mathrm{s}}$ are calculated in two different energy bands $(0.1-4 \mathrm{keV}$ and $4-10 \mathrm{keV})$ and are plotted as a function of the length of the cocoon $l_{\mathrm{c}}(t)$ in Fig. 5: the upper panels refer to $K_{\mathrm{c}}$, while the lower panels refer to $K_{\mathrm{s}}$. In the figure we present the results for all the values of $v$ considered and for a single representative value of the Mach number $M=60$. In each figure the solid curve is for $v=0.1$, the dashed curve is for $v=0.01$ and the dashed-dotted curve for $v=0.001$. Looking at the figure for $K_{\mathrm{c}}(0.1-4 \mathrm{keV})$, we see that, as expected from the results discussed above, in the case of $v=0.1$ the deficit is very low and does not increase with time, instead, the deficit increases at the decreasing of $v$ and shows an increase with time. When we look at the excess emission in the $0.1-4 \mathrm{keV}$ band, measured by $K_{\mathrm{s}}$, we see that the cases $v=0.001$ and $v=0.01$ behave as expected, with the case $v=0.01$ presenting an higher shell emission with values decreasing in time (remember that the shell widens and decreases its density during its evolution). The case $v=0.1$, instead, presents an unexpected behavior. In fact its value of $K_{\mathrm{S}}$ is lower than that found for $v=0.01$ and stays almost constant with time. This however can be understood by considering that the shock in the external medium heats this medium changing its emission properties. The spectral range we are considering for the flux calculation is $0.1-4 \mathrm{keV}$ and the material in the shell may have been heated to a temperature for which the maximum of the emission falls in a harder spectral range. These effects will be however analyzed in more detail in Sect. 4. 

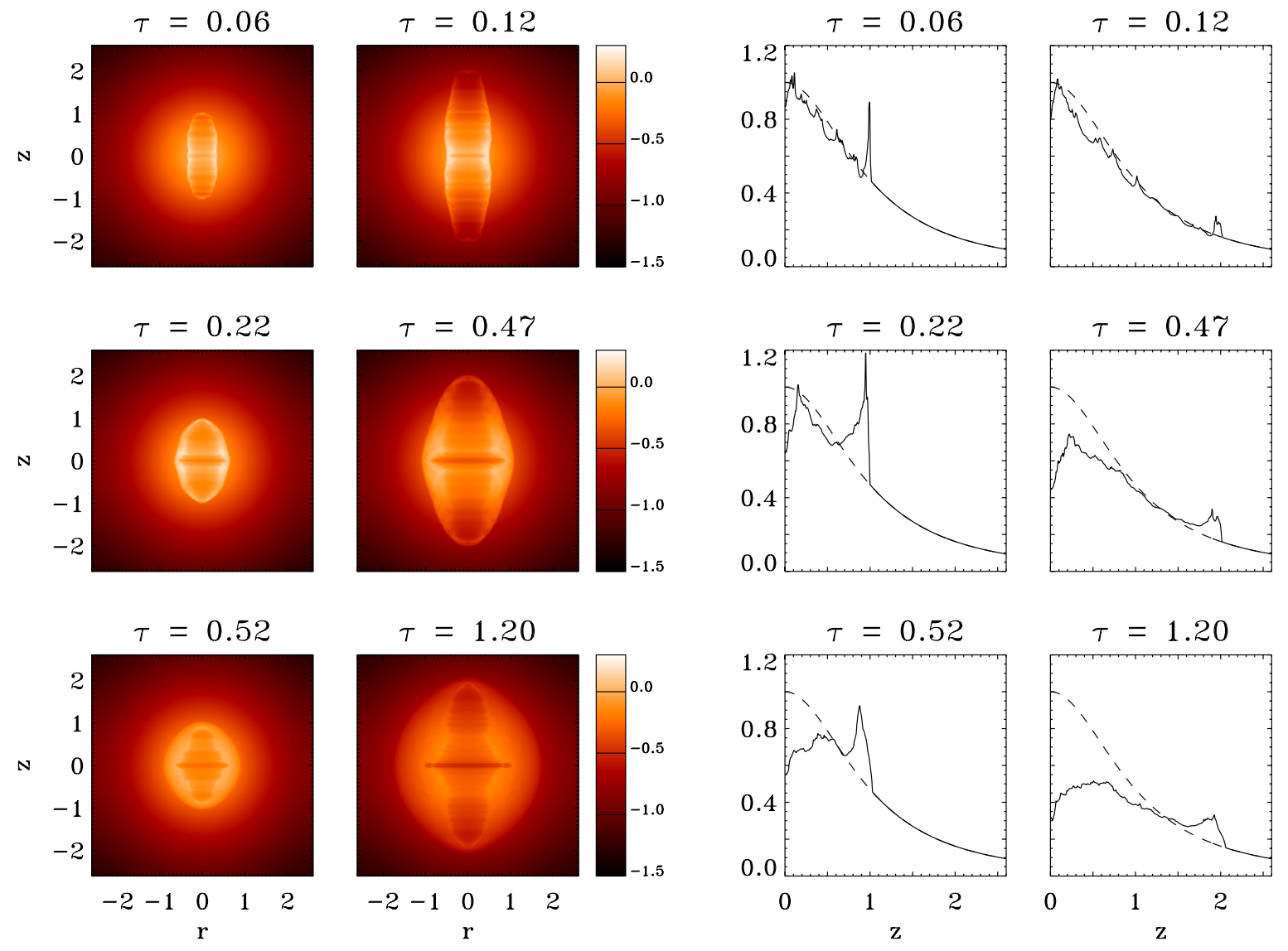

Fig. 2. Simulated X-ray fluxes, in logarithmic scale, in the $0.1-4 \mathrm{keV}$ band for the $M=60$ cases. The rows refer from top to bottom to the $v=0.1,0.01,0.001$ cases respectively. The quantities represented are the same as in Fig. 1.
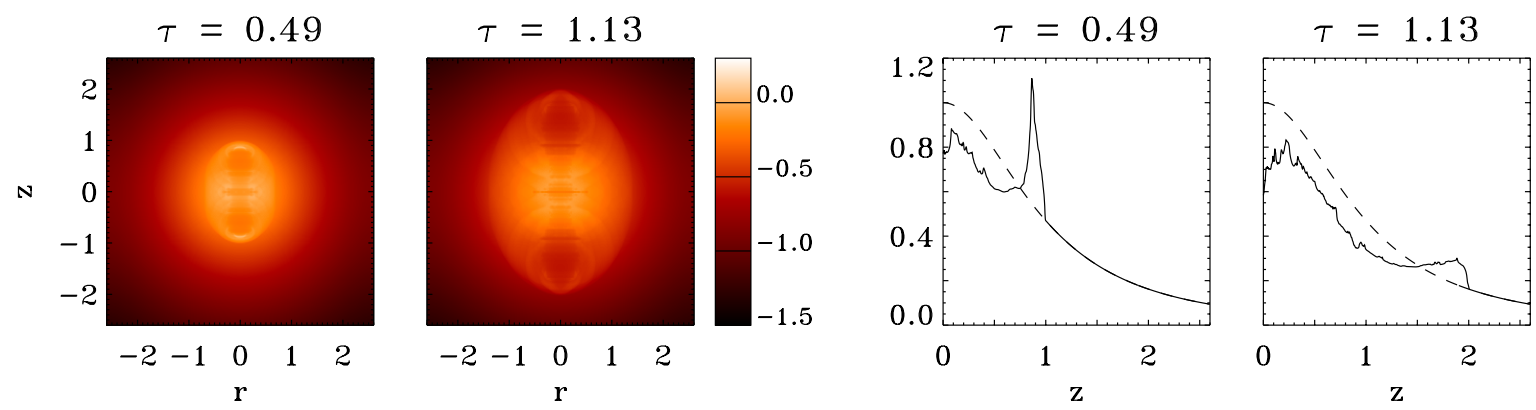

Fig. 3. Simulated X-ray fluxes, in logarithmic scale, in the $0.1-4 \mathrm{keV}$ band for the $M=10, v=0.1$ case. The quantities represented are the same as in Figs. 1, 2.

\section{The shell temperature}

As we discussed, the shock driven in the external medium can heat it and its effects can be more or less evident depending on its strength. The heating of the shell can change the typical emission energies, an example of the consequences of this effect has been shown in the bottom panel of the first column of Fig. 5 where we have seen that the case with $v=0.1$ seemed to present an anomalous behavior. We can now compare the first column of Fig. 5 with the second column where we show the same quantities but in the range $4-10 \mathrm{keV}$. Looking at the bottom panels we see that the case $v=0.1$, which in the softer band presented an excess emission below that of the case $v=0.01$, shows in the harder band an excess emission larger than the other cases. The gas in the compressed shell becomes in fact hotter as we increase the value of $v$ and the emission is then shifted towards higher emission energies. To quantify in more detail this temperature change, we have plotted in the first row of Figs. 6-8 the quantity

$E(T)=\int_{V} \epsilon\left(n, T_{1}, \boldsymbol{x}\right) \delta\left(T_{1}(\boldsymbol{x})-T\right) \mathrm{d}^{3} \boldsymbol{x}$,

where $T_{1}(\boldsymbol{x})$ is the temperature at a given position, $\delta\left(T_{1}-T\right)$ is the Dirac delta function and $\epsilon$ are the radiative losses per unit volume in the $0.1-10 \mathrm{keV}$ band as calculated with the Raymond-Smith code. This quantity measures the energy emitted by the gas at the temperature $T$ in the $0.1-10 \mathrm{keV}$ band. In the first row of Figs. 6, 7 and 8 we have plotted this quantity 

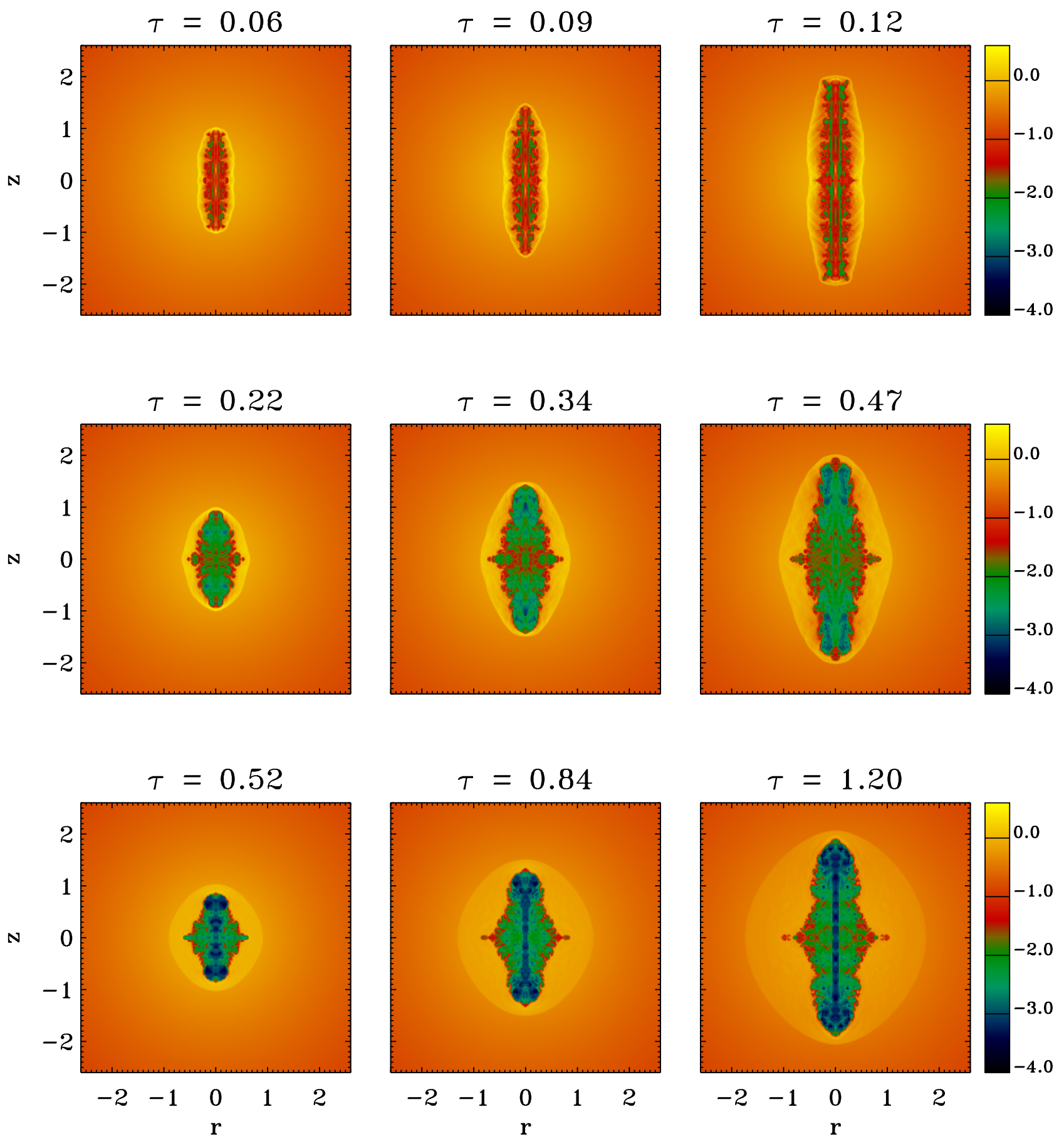

Fig. 4. Density maps for the $M=60$ cases. The scale is logarithmic. The rows refer from top to bottom to the $v=0.1,0.01,0.001$ cases respectively. In the columns density maps are shown at times corresponding to a cocoon length $l_{\mathrm{c}} \sim 1,1.5$ and 2 core radii. The densities are given in unity of the central density of the unperturbed atmosphere.

for the Mach numbers 120, 60 and 10 respectively. In each figure columns are for different values of $v$ and the panels in each column are for the time corresponding to a cocoon length of 2 . In each panel we see a vertical line marking the temperature of the ambient isothermal medium and a peak correponding to the emission from the shocked shell material. We can see that the temperature of the shell increases as we increase the Mach number and the density ratio $v$. Taking into account the cases with the lowest density ratio $v=0.001$ we can see that in the
$M=120$ case the shell temperature is a factor two higher than the ambient one, in the $M=60$ case it is slightly higher while in the $M=10$ case it is clear that there is emission also from gas with a temperature lower than the ambient one. This fact agrees with several observations (e.g. Perseus A) in which the coolest gas is found in the shell of enhanced emission. The cooling in this simulation is due to the adiabatic expansion of the shell after being compressed by a weak shock. As time elapses, radiative losses may become important and contribute to the cooling 

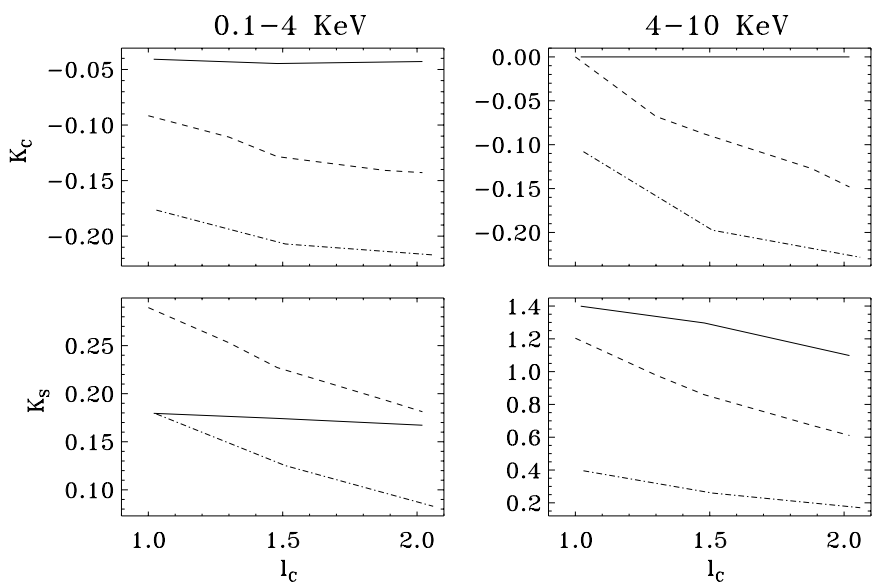

Fig. 5. (Upper panels) Plot of the integrated flux deficit as a function of cocoon length in the $0.1-4 \mathrm{keV}$ band (left) and in the 4-10 keV band (right) for the $M=60$ cases. This quantity is defined in Eq. (9). (Lower panels) Plot of the excess emission as a function of cocoon length in the $0.1-4 \mathrm{keV}$ band (left) and in the $4-10 \mathrm{keV}$ band (right) for the $M=60$ cases. This quantity is defined in Eq. (10). In the four panels the three lines refer to the $v=0.1$ (solid), $v=0.01$ (dashed) and $v=0.001$ (dash-dotted) cases.
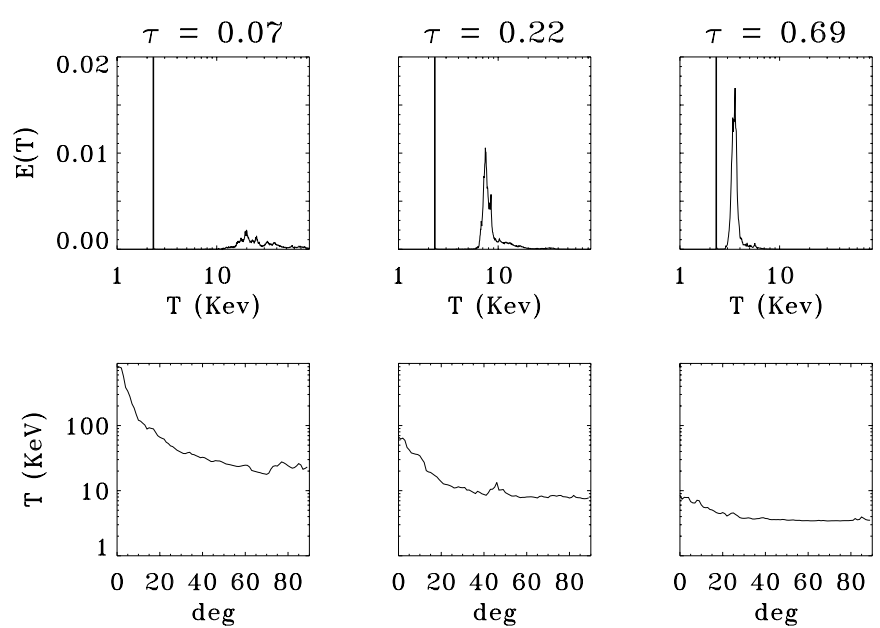

Fig. 6. Temperature plots for the $M=120$ cases $(v=0.1,0.01,0.001$ from left to right respectively) at a time corresponding to a cocoon length of 2 core radii. (Upper panels) Plot of the quantity $E(T)$ as defined in Eq. (11). It represents the energy emitted in the $0.1-10 \mathrm{keV}$ band by the gas at the temperature $T$. The vertical line marks the temperature of the ambient isothermal medium while the peak correponds to the compressed shell emission. The emission is normalized to the total emission from the unperturbed atmosphere. (Lower panels) Plot of the shell average temperature as a function of the angle with origin in $r=0, z=0$. The $0^{\circ}$ angle corresponds to the direction of the jet axis. The ambient temperature is taken $2.3 \mathrm{keV}$.

of the shell: for a central density of $0.04 \mathrm{~cm}^{-3}$ and a temperature of $3 \mathrm{keV}$ (e.g. Perseus A, see Schmidt et al. 2002), the radiative cooling time is $\sim 3 \times 10^{7}$ years, i.e. close to the simulated time of the $M=10, v=0.1$ case. The shell temperature can reach temperature up to $800 \mathrm{keV}$ for the case $M=120$ and $v=0.1$. The heating of the shell is not uniform but depends on the shock strength and it is higher towards the jet head and becomes lower further from the head. This is represented in
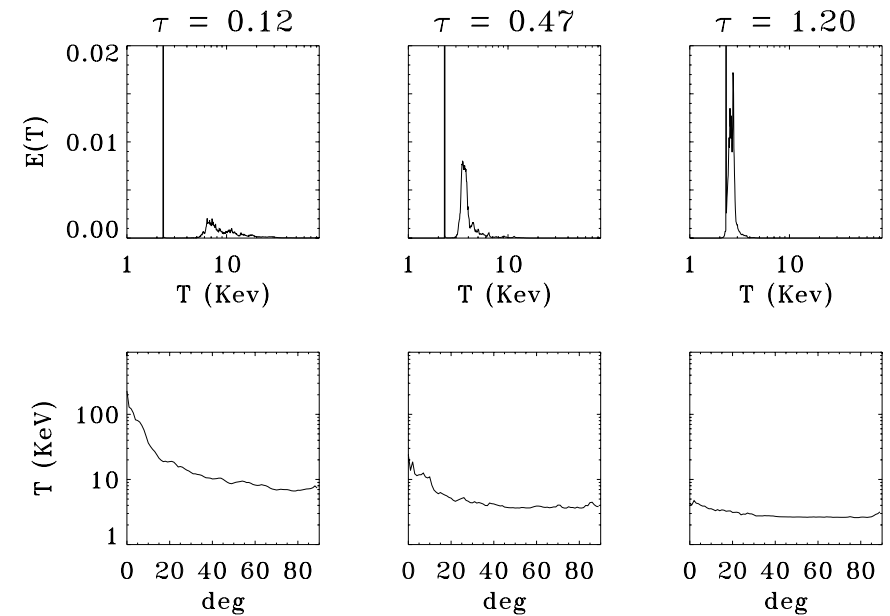

Fig. 7. Temperature plots for the $M=60$ cases $(v=0.1,0.01,0.001$ from left to right respectively) at a time corresponding to a cocoon length of 2 core radii. The quantities plotted are the same as in Fig. 6.
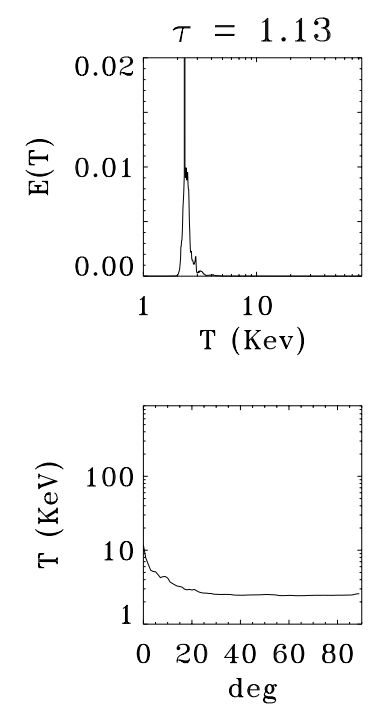

Fig. 8. Temperature plots for the $M=$ $10, v=0.1$ case at a time corresponding to a cocoon length of 2 core radii. The quantities plotted are the same as in Figs. 6 and 7.

the second row of Figs. $6-8$, where we show the average shell temperature as a function of angle for all the cases considered.

From the figures we see that, for the cases with $v=0.1$ and $M=60,120$, the shell temperature is everywhere larger than $8 \mathrm{keV}$ and reaches temperatures up to $850 \mathrm{keV}$ at the jet head of the $M=120$ case. The lower density cases, instead, present an increase in temperature mainly concentrated in the forward part of the cocoon. This temperature distribution will have consequences in the shell morphology as seen in different $\mathrm{X}$-ray bands. In fact, we expect to see emission from the forward part of the cocoon at high energies, while the backward part is expected to be more dominant at lower energies.

\section{The cocoon dynamics}

The numerical simulations presented in Sects. 2-4 have shown that the expanding cocoon drives a shock in the ambient medium, which is then compressed and heated. The amount of compression and heating is fundamental in determining the $\mathrm{X}$-ray emission properties. The results we have shown tell us 
that, as also discussed by Reynolds et al. (2001), the strength of the shock, driven by the cocoon, weakens as the cocoon expands and the stage at which the transition between a strong and a weak shock occurs depends on the jet properties, i.e. its Mach number and its density ratio. In this section we will try to determine in a more quantitative way this dependence of the transition on the jet physical properties. We will do that through a comparison between analytical models for the cocoon expansion in an homogeneous medium and the results of our numerical simulations. In Sect. 5.1 we will then examine the cocoon dynamics in a uniform medium, in Sect. 5.2 we will examine the dynamics in a stratified medium and in Sect. 5.3 we will try to determine the dependence of the transition discussed above on the jet properties.

\subsection{Expansion in a uniform medium}

The first attempt to build an analytical model for the cocoon dynamics is due to Begelman \& Cioffi (1989) and Cioffi \& Blondin (1992). They consider only the case of strongly overpressured cocoons, for which they consider only strong shocks driven in the external medium and they essentially neglect the external pressure. For our purposes, we have to extend the description to a more general situation, in which the external shock can be of any strength and the external pressure is taken into account. We describe in detail the model in the Appendix, here we give only the resulting behavior of the extended cocoon radius and cocoon pressure versus time: the cocoon radius is given by

$r_{\mathrm{e}}^{2}=c_{\mathrm{se}}^{2} t^{2}+\sqrt{\gamma^{2}-1} r_{\mathrm{j}} v^{1 / 4} M c_{\mathrm{se}} t$

and the cocoon pressure is

$\frac{P_{\mathrm{c}}}{P_{\mathrm{ext}}}=1+\frac{\gamma(\gamma-1)}{2}\left(\frac{1}{v^{1 / 2} M^{2}} \frac{c_{\mathrm{se}}^{2} t^{2}}{r_{\mathrm{j}}^{2}}+\frac{\sqrt{\gamma^{2}-1}}{v^{1 / 4} M} \frac{c_{\mathrm{se}} t}{r_{\mathrm{j}}}\right)^{-1}$.

Equations (12), (13) describe two different phases in the evolution: initially the cocoon is strongly overpressured and the solutions behave in the same way as described by the Begelman \& Cioffi (1989) model, with the radius proportional to $t^{1 / 2}$ and the pressure proportional to $t^{-1}$. As the pressure decreases, the contribution of the external pressure becomes more important and the external shock becomes weak. In this second phase the pressure tends to become constant and the extended cocoon expands essentially at the sound speed and increases as $\propto t$. We can use Eq. (A.4) to determine the radius of the cocoon proper: in the first phase the internal radius increase $\propto t^{1 / 2}$ like the external one not allowing the shell to expand, while in the second phase it tends to a constant value. The compression of the external medium can be related to the relative shell thickness and we can observe that, while it stays constant in the first phase, as already discussed above, it tends to increase in the second phase. The transition between these two phases happens when the two terms on the right hand side of Eq. (12) become comparable; then, assuming a cocoon length $l_{\mathrm{c}}=v^{1 / 2} v_{\mathrm{j}} t$, we can determine a scaling law for the length of transition $l_{\mathrm{c}}^{*}$ between the two regimes

$l_{\mathrm{c}}^{*} \propto M^{2} v^{3 / 4}$.
Therefore, for a given Mach number and for a given length of the cocoon, we expect the jets with lower density ratios to be less overpressured and therefore to form wider and less dense shells.

In a similar way we can also find a solution assuming a spherical symmetry for the cocoon, that is solving the system of Eqs. (A.6-A.8) taking $V_{\mathrm{c}}=4 \pi / 3 r_{\mathrm{e}}^{3}$. Taking into account separately the strongly overpressured $\left(P_{\mathrm{c}} \gg P^{*}\right)$ and the weakly overpressured $\left(P_{\mathrm{c}} \simeq P^{*}\right)$ phases we can find two distinct regimes of expansion: a supersonic one during which the external radius behaves like

$r_{\mathrm{e}}=\left[\frac{25\left(\gamma^{2}-1\right)}{9 \pi}\right]^{1 / 5}\left(\frac{L_{\mathrm{j}}}{\rho_{\mathrm{ext}}}\right)^{1 / 5} t^{3 / 5}$

and the pressure decreases as

$P_{\mathrm{c}}=\frac{3(\gamma-1)}{4 \pi}\left[\frac{9 \pi}{25\left(\gamma^{2}-1\right)}\right]^{3 / 5}\left(\rho_{\mathrm{ext}}^{3} L_{\mathrm{j}}^{2}\right)^{1 / 5} t^{-4 / 5}$

and a weakly overpressured phase during which the cocoon pressure tends to a constant value and the radius $r_{\mathrm{e}}$ expands $\propto t$ at the sound speed. Assuming spherical symmetry also for the cocoon proper $\left(V_{\mathrm{ci}}=4 \pi 3 r_{\mathrm{i}}^{3}\right)$ we can derive the behavior of the cocoon radius $r_{\mathrm{i}}$ solving Eq. (A.5): $r_{\mathrm{i}}$ expands as $t^{3 / 5}$ during the strongly overpressured phase, with the same rate of expansion of $r_{\mathrm{e}}$, while it behaves as $t^{1 / 3}$ during the weakly overpressured one. As in the cylindrical geometry model the shell is allowed to widen only in the weakly overpressured phase. We can then determine a scaling law for the radius of transition between the two regimes imposing that the expansion speed determined by Eq. (15) approaches the sound speed $c_{\mathrm{se}}$

$r_{\mathrm{e}}^{*} \propto\left(\frac{L_{\mathrm{j}}}{c_{\mathrm{se}}^{3} \rho_{\mathrm{ext}}}\right)^{1 / 2}$.

In the spherical symmetry model the cocoon properties scale with the jet power since it is the only jet parameter that enters the system of equation solved in the Appendix. It is worthwhile noticing that our solution for the spherical strongly overpressured cocoon (Eqs. (15) and (16)) is the same, apart from numerical constants, of Heinz et al. (1998) who solved a system of equations similar to ours but limited their solution to the strongly overpressured regime.

Looking at the geometry and at the detailed structure of the shell and the cocoon, these analytical models are too simplified to describe in detail the radiative properties of the cocoon but still they show clearly how the relative thickness of the shell can increase only in a weakly overpressured regime.

\subsection{Expansion in a stratified medium}

The cocoon expansion in a stratified medium is, of course, much more complicated, since the external pressure is not constant, and the development of an analytical model becomes more problematic. However the above discussion can provide a framework for understanding the results of numerical simulation.

We recall that the presence of a brightness depression depends substantially on the relative thickness of the shell. 

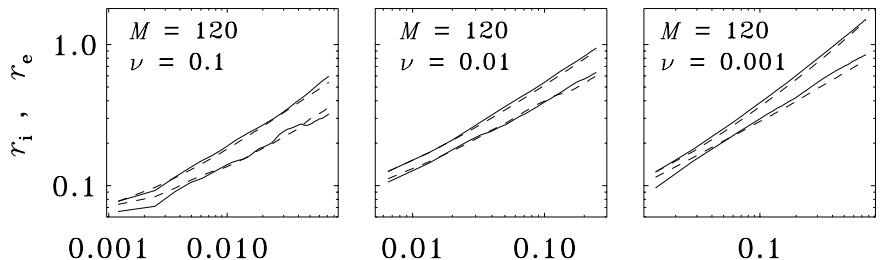

0.1
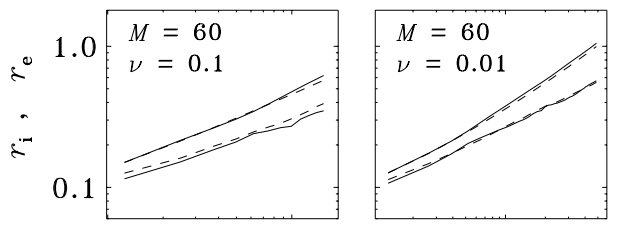

0.1

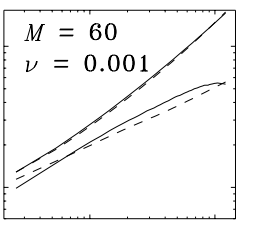

0.1

1.0

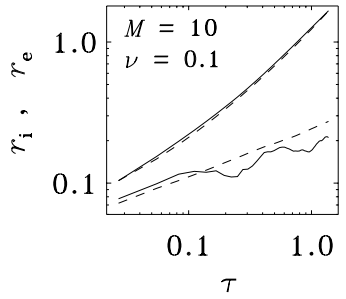

Fig. 9. Plot of the internal and external radii vs time. The rows refer to the different Mach cases while columns to the different $v$ values. The solid lines show the extended and internal cocoon radii as determined in the simulations taking an average value between $1 / 8$ and $1 / 4$ of the total length of the cocoon. The dashed lines show the same quantities as determined by our analytical model assuming that the expansion of the cocoon is driven by the average pressure of the cocoon.

We then must first study the evolution of the extended cocoon radius and the cocoon proper one. We first analyze the behavior of the extended cocoon radius $r_{\mathrm{e}}$ by comparing the results directly obtained by the numerical simulations with an estimate obtained, using the average cocoon pressure as the driver for the expansion. More precisely, we obtain this estimate by integrating numerically Eq. (A.8), where for $P_{\mathrm{c}}(t)$ we use the average value obtained at every time step from the simulations. For defining the extended cocoon radius in the simulations, we concentrate on the base portion of the cocoon and we take an average value of the bow shock radius between $1 / 8$ and $1 / 4$ of the total length of the cocoon. The results of the comparison are represented in Fig. 9, where we plot $r_{\mathrm{e}}$ and $r_{\mathrm{i}}$ as a function of time for all the cases we have considered. In all the panels the solid curves correspond to the results obtained in the simulations, while the dashed curves correspond to the estimates obtained through the average pressure. The figures show that this estimate reproduces very well the actual behavior of the extended cocoon radius: this result tells us that the average cocoon pressure is a good estimate of the local pressure driving the cocoon expansion. In a similar way we can proceed for the cocoon radius $r_{\mathrm{i}}$, for which we can use Eq. (A.5) where with $\eta L_{\mathrm{j}}$ we intend the fraction of jet power that is thermalized and goes into internal energy of the cocoon. This fraction can be estimated from Eq. (A.6). Equation (A.4) can be integrated giving

$V_{\mathrm{c}}=\frac{1}{P_{\mathrm{c}}(t)^{1 / \gamma}}\left\{P_{\mathrm{c}}(0)^{1 / \gamma} V_{\mathrm{c}}(0)+\frac{\gamma-1}{\gamma} \int_{t_{0}}^{t} \eta L_{\mathrm{j}} P_{\mathrm{c}}^{(1-\gamma) / \gamma} \mathrm{d} t\right\}$
Table 2. The table shows the exponent $\alpha$ of the fitted power laws $\tau^{\alpha}$ for the quantities $l_{\mathrm{c}}$ (cocoon length), $r_{\mathrm{e}}$ (extended cocoon radius) and $r_{\mathrm{i}}$ (internal cocoon radius). The left column refers to the initial phase of the evolution of the cocoon $\left(l_{\mathrm{c}}<1.0\right)$ while the second one to the advanced one $\left(l_{\mathrm{c}}>1.5\right)$

\begin{tabular}{ccc}
\hline \hline$v=0.1$ & $l_{\mathrm{c}}<1.0$ & $l_{\mathrm{c}}>1.5$ \\
\hline$l_{\mathrm{c}}$ & 0.85 & 0.85 \\
$r_{\mathrm{e}}$ & 0.54 & 0.67 \\
$r_{\mathrm{i}}$ & 0.43 & 0.56 \\
\hline \hline$v=0.01$ & $l_{\mathrm{c}}<1.0$ & $l_{\mathrm{c}}>1.5$ \\
\hline$l_{\mathrm{c}}$ & 0.75 & 0.95 \\
$r_{\mathrm{e}}$ & 0.56 & 0.71 \\
$r_{\mathrm{i}}$ & 0.45 & 0.53 \\
\hline \hline$v=0.001$ & $l_{\mathrm{c}}<1.0$ & $l_{\mathrm{c}}>1.5$ \\
\hline$l_{\mathrm{c}}$ & 0.77 & 0.89 \\
$r_{\mathrm{e}}$ & 0.69 & 0.81 \\
$r_{\mathrm{i}}$ & 0.46 & 0.07 \\
\hline
\end{tabular}

where again for $P_{\mathrm{c}}$ we can use the average cocoon pressure obtained above. This equation gives us an estimated behavior of the cocoon volume and from this we can estimate the behavior of the cocoon radius as $r_{\mathrm{i}}=\sqrt{V_{\mathrm{c}} / K l_{\mathrm{c}}}$ where $K$ is a form factor that depends on the shape of the cocoon. One problem in determining $r_{\mathrm{i}}$ is that the shape of the cocoon is strongly variable since the interface between cocoon and external shocked material is subject to Kelvin-Helmholtz instabilities. Moreover, the shape of the cocoon may depend on the jet physical parameters. However, we can see from Fig. 9 (where, as before with $r_{\mathrm{e}}$, we compare this estimate with the actual results obtained from the simulations) that using an appropriated average values for $K$, we can capture quite well the average behavior. The form factor should be $K=\pi$ for a cylinder and $K=2 \pi / 3$ for a hemisphere. The value that can be estimated from the simulations and that works quite well for every case is $K \sim 2$. Again, this good agreement between the estimates obtained through the usage of the average cocoon pressure and the actual results tells us that we can use the average pressure behavior for understanding the cocoon dynamics.

Looking in more detail at the behavior of the extended cocoon radius, we observe that, as expected from the discussion of the uniform case, its expansion velocity decreases faster initially, when the cocoon is strongly overpressured, and then decreases more slowly, when the shock becomes weaker. Fitting a power law $\propto \tau^{\alpha}$ for the quantities $l_{\mathrm{c}}, r_{\mathrm{e}}, r_{\mathrm{i}}$ in the initial and terminal part of the evolution for the cases with $M=60$, we get the exponents $\alpha$ reported in Table 2. We see that, at later times, the exponents of $r_{\mathrm{e}}$ become systematically larger than those obtained at the initial times. Moreover we see that the differences between the two exponents are larger for the lower density cases and for $v=0.1$ are minimal. In this last case, in fact the cocoon stays strongly overpressured during the whole evolution.

A similar analysis can be done for the cocoon radius $r_{\mathrm{i}}$. We see that in the high and intermediate density cases the exponent 
for $r_{\mathrm{i}}$ also increases but less than the exponent for $r_{\mathrm{e}}$. In the low density case, instead, $r_{\mathrm{i}}$ becomes almost constant in time. This behavior tells us that, when the cocoon is not any more strongly overpressured, the relative shell thickness start to increase as it happened in the homogeneous case. The behavior of the relative thickness of the shell can be in fact derived from Table 2, since it is related to the ratio $r_{\mathrm{i}} / r_{\mathrm{e}}$. We then see that it has in general a slower increase at the beginning of the evolution, and accelerates in the following phases. As discussed above, the effects of not being strongly overpressured are more evident by decreasing the value of $v$ and, in fact, the increase of the relative cocoon width is larger for smaller $v$. If we compare the cocoon widths for equal distances of jet propagation we have a further effect that amplify the consequences of the behavior discussed above, namely we have to take into account that the advance velocity of the head of the jet decreases when we decrease the value of $v$.

In this section we have seen that the transition from a strongly overpressured cocoon to a cocoon which is essentially in pressure equilibrium with the surroundings leads to different properties of the shell of the compressed external material. In Sect. 5.3 we will try to see whether it is possible to determine in a more quantitative way how this transition depends on the jet physical parameters.

\subsection{The transition from strong to weak shocks}

From the above discussion we have seen that the average cocoon pressure can be used for interpreting the cocoon dynamics and therefore determining the transition between the strong and weak shock regimes. In the case of uniform external medium, the cocoon pressure, after a decrease proportional to $t^{-1}$, tends to a constant. In the present case, with a decreasing external pressure, we expect that the cocoon pressure does not tend to a constant but that it will tend to follow the behavior of $P^{*}$, defined in Eq. (A.7).

In Fig. 10 we have plotted the behavior of $P_{\mathrm{c}}$ (solid curve) and $P^{*}$ (dashed curve) against the cocoon length, where $P_{\mathrm{c}}$ is the average cocoon pressure and $P^{*}$ is the external pressure averaged over the cocoon volume. The ratio between the two quantities gives a measure of the average strength of the shock driven in the external medium. Looking at the behavior of the cocoon evolution in the different cases we have an always strongly overpressured expansion for the cases $M=120$ and $v=0.1,0.01$ and for $M=60$ and $v=0.1$, in these three cases the transition length would be larger than the actual longitudinal size of our domain; two cases showing a transition from strongly overpressured to weakly overpressured cocoons for $M=120, v=0.001$ and $M=60, v=0.01$; finally we have two cases where the cocoon is always weakly overpressured, i.e. for $M=60, v=0.001$ and $M=10, v=0.1$. This is in agreement with the discussion on the X-ray flux distributions following Figs. 1-3.

In order to determine the length of the cocoon at which the transition occurs, we look for a scaling law for the quantity $\left(P_{\mathrm{c}}-P^{*}\right) / P^{*}$ as a function of the parameters $l_{\mathrm{c}}, M$ and $v$ during the initial strongly overpressured phase. Taking into account

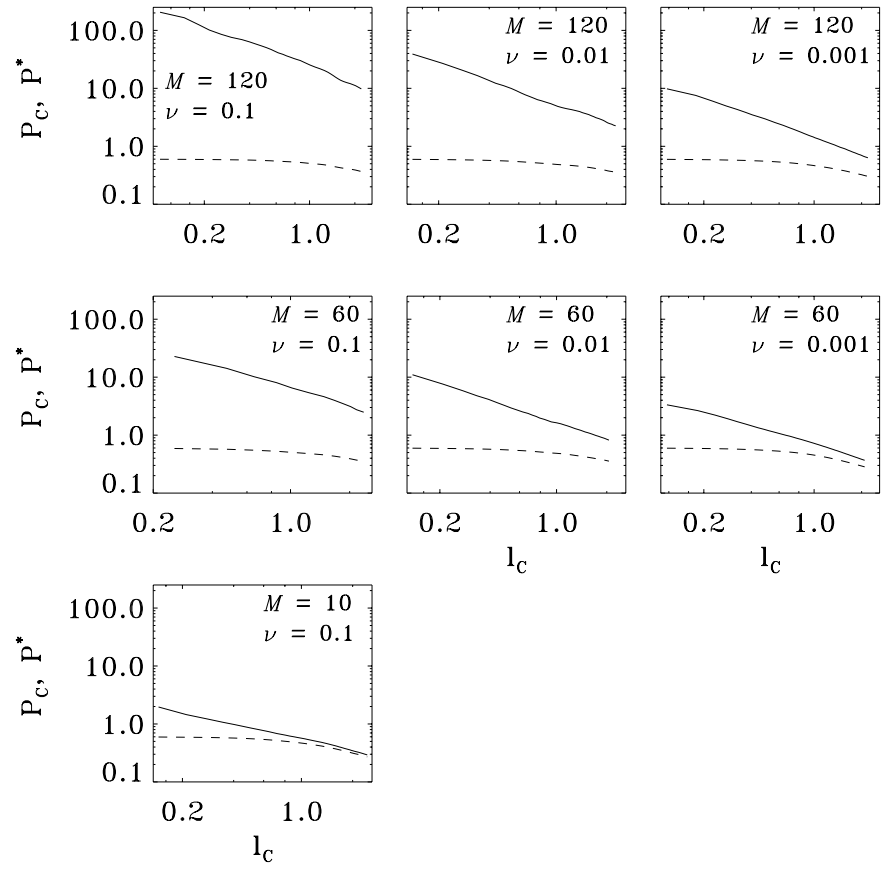

Fig. 10. Plot of the pressure vs. cocoon length. The rows refer to the different Mach numbers while columns to the different $v$ values. The solid line shows the evolution of the average cocoon pressure with time in the different cases. The dashed one represents the external pressure averaged on the cocoon volume as defined in Eq. (A.7).

only the cases that are stronlgy overpressured at the beginning of the evolution, we notice that the initial decrease of the average cocoon pressure follows a similar behavior for all the cases that we have considered: with a power law fit $\left(P_{\mathrm{c}}-P^{*}\right) / P^{*} \propto l_{\mathrm{c}}^{-\alpha}$ to the initial evolution of the different cases we find a mean value $\alpha=0.9$ with an uncertainness of $10 \%$. In Table 3 we show the values of the quantity $\left(P_{\mathrm{c}}-P^{*}\right) / P^{*}$ at the beginning of the evolution, when the cocoon length is one third the core radius and the cocoon is typically strongly overpressured (notice that the cases $M=10, v=0.1$ and $M=60, v=0.001$ make an exception). These values can be represented, with good approximation by the scaling $\left(P_{\mathrm{c}}-P^{*}\right) / P^{*} \propto M^{1.85} v^{0.62}$. The general scaling law thus becomes:

$\frac{P_{\mathrm{c}}-P^{*}}{P^{*}} \propto l_{\mathrm{c}}^{-0.9} M^{1.85} v^{0.62}$

The scaling relation (19) shows that the initial pressure of the cocoon is not proportional to an arbitrary combination of the jet parameters $M$ and $v$ but it scales with the normalized kinetic jet power $L_{\mathrm{j}} / L_{\mathrm{k}}=M^{3} v$ (see Eq. (7)) as $\left(P_{\mathrm{c}}-P^{*}\right) / P^{*} \propto$ $l_{\mathrm{c}}^{-0.9}\left(L_{\mathrm{j}} / L_{\mathrm{k}}\right)^{0.62}$. This result is confirmed by the plot of the values of $\left(P_{\mathrm{c}}-P^{*}\right) / P^{*}$ reported in Table 3 against the jet kinetic luminosity $L_{\mathrm{j}} / L_{\mathrm{k}}$, after setting $l_{\mathrm{c}}=0.3$. The plot is shown in Fig. 11.

Of course the transition from a strongly to a weakly overpressured regime is not sudden, but gradually happens when $\left(P_{\mathrm{c}}-P^{*}\right) / P^{*}$ becomes of the order of unity. Setting a constant value for $\left(P_{\mathrm{c}}-P^{*}\right) / P^{*}$ of this order in Eq. (19) we derive the 


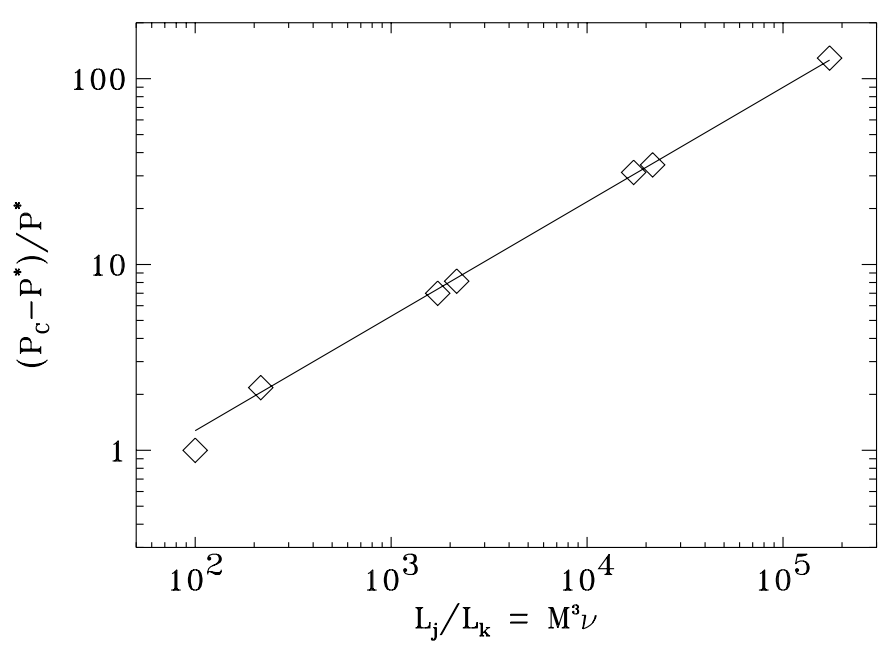

Fig. 11. Plot of the values of $\left(P_{\mathrm{c}}-P^{*}\right) / P^{*}$ at a cocoon length $l_{\mathrm{c}}=0.3$ (see Table 3) against jet kinetic luminosity $L_{\mathrm{j}} / L_{\mathrm{k}}=M^{3} v$. The solid line is a $\left(P_{\mathrm{c}}-P^{*}\right) / P^{*} \propto\left(L_{\mathrm{j}} / L_{\mathrm{k}}\right)^{0.62}$, that is also the best fit, excluding the value of the case $M=10, v=0.1$ that is in a weakly overpressured regime already.

scaling law for transition length $l_{\mathrm{c}}^{*}$ (as we have done for the uniform case, see Eq. (14)).

$l_{\mathrm{c}}^{*} \propto M^{2.05} v^{0.69}$

or in terms of the jet kinetic power

$l_{\mathrm{c}}^{*} \propto\left(\frac{L_{\mathrm{j}}}{L_{\mathrm{k}}}\right)^{0.69}$.

Equation (20) represents a family of curves in the plane $(M, v)$, and, for a given cocoon length one of the curves of this family will mark the separation between cocoons that drive strong shocks in the ambient medium (strongly overpressured regime) and cocoons that drive only weak shocks (weakly overpressured regime). In Sect. 6 we will discuss the astrophysical relevance of these results.

\section{Discussion}

In Sects. 3-5 we have seen that strongly and weakly overpressured cocoons present different X-ray morphologies. The former ones will show no deficit of emission accompanied by a strong emission from a shell marking the shock, driven by the cocoon expansion. The material in the shell will be much hotter than the ambient medium, the shell emission will then be shifted to higher frequencies and therefore more visible at higher X-ray energies, especially near the jet head. Weakly overpressured cocoons will be instead characterized by the presence of a deficit of emission in the cocoon, while the emission from the shell will be much less visible than in the previous case; in addition, the material in the shell will be essentially at the same temperature as the ambient medium and there will be no change in the emission spectrum. These two regimes depend on the jet physical parameters and on the age of the cocoon. High Mach number jets with high densities will be
Table 3. Values of the quantity $\left(P_{\mathrm{c}}-P^{*}\right) / P^{*}$ for the different simulations evaluated at a cocoon length $l_{\mathrm{c}}=0.3$.

\begin{tabular}{cccc}
\hline \hline$M \backslash v$ & 0.1 & 0.01 & 0.001 \\
\hline 120 & 192.03 & 31.30 & 6.99 \\
60 & 34.35 & 8.13 & 2.17 \\
10 & 0.99 & & \\
\hline
\end{tabular}

strongly overpressured for a longer fraction of their life, decreasing the density and the Mach number the transition to weakly overpressured cocoon will occur at an earlier stage. We have found that the scaling of the transition length from one regime to the other is given to a good approximation by Eq. (20). Fixing a length of the cocoon, from this equation we can then obtain a relation between Mach number and density ratio such as $M=C\left(l_{\mathrm{c}}\right) v^{-1 / 3}$. This scaling law has been represented in Fig. 12 with the dotted, dashed and dash-dotted lines for cocoon lengths equal to $0.5,1$ and 2 core radii respectively. The proportionality constant $C\left(l_{\mathrm{c}}\right)$ has been set to fulfill the transition conditions in the cases where the transition between the two regimes is observed, i.e. $M=60, v=0.01$ and $M=120, v=0.001$. In the same figure we can also notice the presence of another line, that individuates a region in which the jet becomes subsonic with respect to its internal sound speed.

Since Mach number and density ratio are physically very meaningful parameters, but cannot be determined for actual jets, it is favorable to express the scaling relation in terms of the jet kinetic power. In order to do so, we make use of Eq. (21) where $L_{\mathrm{k}}$ is a quantity that depends on the properties of the environment. From this relation we see that jets with the same kinetic power have the same transition length between the two phases. The curves in Fig. 12 separating the strongly and weakly overpressured regimes, which are given by $M \propto v^{-1 / 3}$, will therefore correspond also to a constant value of $L_{\mathrm{j}} / L_{\mathrm{k}}$. These considerations tell us that the separation between the two regimes is essentially determined by the jet kinetic power: the transition will occur at higher values of $l_{\mathrm{c}}$ for high kinetic power jets and at lower $l_{\mathrm{c}}$ for low kinetic power jets. For instance the dashed curve shown in Fig. 12 representing the separation between the two regimes for a cocoon length equal to 2 core radii corresponds to $L_{\mathrm{j}} / L_{\mathrm{k}} \sim 4.7 \times 10^{3}$. The properties of the environment enter in the parameter $L_{\mathrm{k}}$ that fixes a measure for the jet kinetic power. For a typical cluster environment with a central density $10^{-2} \mathrm{~cm}^{-3}$, a core radius $100 \mathrm{kpc}$ and a temperature $3 \mathrm{keV}$ we have $L_{\mathrm{k}}=6 \times 10^{42} \mathrm{erg} \mathrm{s}^{-1}$ and the dividing kinetic power for a cocoon length equal to 1.5 core radii will be $L_{\mathrm{j}} \sim 1.9 \times 10^{46} \mathrm{erg} \mathrm{s}^{-1}$. It is important to notice that for jets that are slightly supersonic with respect to their internal sound speed the jet enthalpy flux becomes comparable to the kinetic one. In these cases our estimates for the jet kinetic power should be corrected at most by a factor two in order to obtain an approximation of the total power of the jet.

Considering the particular case of Cygnus A, its cluster environment is characterized by a central density $0.07 \mathrm{~cm}^{-3}$, 


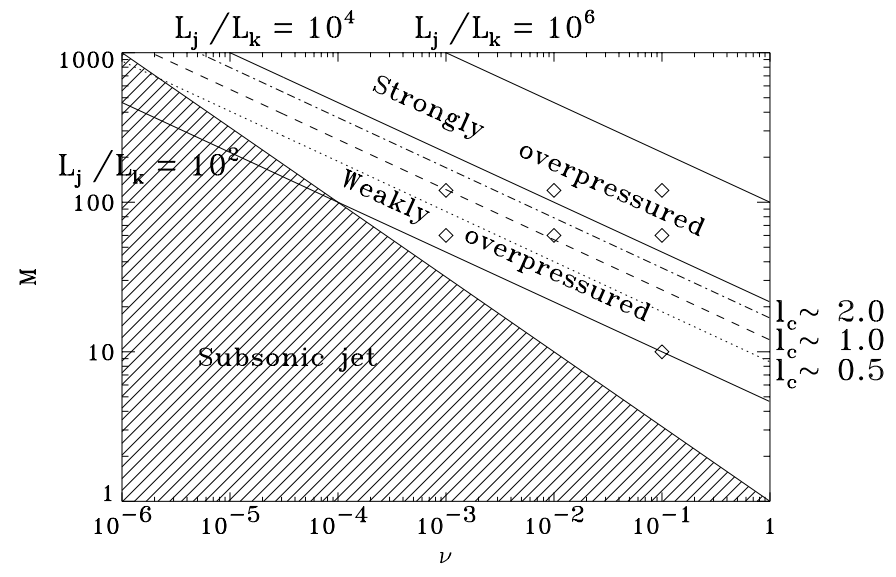

Fig. 12. Representation of the different regimes of the cocoon expansion in the $M-v$ plane. Inside the dashed area the jet becomes subsonic with respect to its internal sound speed. The solid lines correspond to a constant kinetic jet power $L_{\mathrm{j}} / L_{\mathrm{k}}=M^{3} v$ given in unit of the power $L_{\mathrm{k}}$ defined in Eq. (5). The fragmented curves separate strongly and weakly overpressured regimes for different lengths of the cocoon given in core radii units. It can be seen that these lines correspond to lines of constant kinetic jet power. The diamonds correspond to the simulated cases.

a core radius $35 \mathrm{kpc}$ and a temperature $3.4 \mathrm{keV}$ (Carilli et al. 1994), giving $L_{\mathrm{k}}=6.2 \times 10^{42} \mathrm{erg} \mathrm{s}^{-1}$. The radio lobes of Cygnus A show an extent of $\sim 70 \mathrm{kpc}$, twice the core radius $\left(l_{\mathrm{c}}=2\right)$. For this length the dividing power is given by $L_{\mathrm{j}} / L_{\mathrm{k}} \sim 4.7 \times 10^{3}$, as it is possible to see in Fig. 12. Since Cygnus A clearly shows deficit of X-ray emission and therefore is in a weakly overpressured regime, these estimates give an upper limit to the kinetic power of its jet $\sim 2.9 \times 10^{46} \mathrm{erg} \mathrm{s}^{-1}$. Nevertheless the pressure of the expanding cocoon must be higher than the ambient one since observations by Chandra (Smith et al. 2002) show clearly that the shell is slightly hotter than the surrounding medium. Then the cocoon is still expanding as a (weak) shock wave more than a sound wave.

Another well known example of the interaction between a radio source and the thermal gas of a cluster is the FR I type radio galaxy $3 \mathrm{C} 84$ inside the Perseus cluster. The cluster core can be modeled with a central electron density $0.04 \mathrm{~cm}^{-3}$, a core radius $a \sim 50 \mathrm{kpc}$ and a central temperature $T \sim 3.1 \mathrm{keV}$ (Schmidt et al. 2002) yielding $L_{\mathrm{k}}=6 \times 10^{42} \mathrm{erg} \mathrm{s}^{-1}$. The radio lobes show an average extent of $\sim 22 \mathrm{kpc}$, giving a dividing power $L_{\mathrm{j}} / L_{\mathrm{k}} \sim 5.2 \times 10^{2}$. These estimates give an upper limit on the kinetic luminosity of the jet $L_{\mathrm{j}}=3.1 \times 10^{45} \mathrm{erg} \mathrm{s}^{-1}$. This upper limit can be lowered considering that the shell of enhanced emission surrounding the cavities contains clearly the $\mathrm{X}$-ray coolest gas in the cluster (Fabian et al. 2001). This fact rules out the presence of strong shocks driven by the expanding cocoon. In our scheme this means that the cocoon must be in a weakly overpressured regime in the first phase of expansion yet as for example in the case $M=10, v=0.1$. As it can be seen in Fig. 8 the average temperature of the shell for this simulation is equal to the ambient one and in the shell there is also emission from gas cooler than the ambient one. This cooling is due to the adiabatic expansion of the shell after being compressed by a weak shock, in a way similar to that described in the simulations of expanding hot bubbles by Brighenti \& Mathews (2002). Taking this case $\left(L_{\mathrm{j}} / L_{\mathrm{k}}=100\right)$ to determine a limit on the kinetic power of the jet we obtain $L_{\mathrm{j}}=6.3 \times 10^{44} \mathrm{erg} \mathrm{s}^{-1}$. For deriving the total jet power this estimate should be corrected by the enthalpy term, that for the above values of $M$ and $v$ is approximately equal to the $30 \%$ of the kinetic power. This estimate is similar to the one by Fabian et al. (2001) who used the analytical bubble model by Churazov et al. (2000) to determine their limits. This substantial agreement can be understood considering that the Churazov et al. (2000) model solves exactly Eq. (A.4) with spherical symmetry assuming that the bubble pressure is equal to the external one. The limits of Fabian et al. (2001) are then determined requiring that the bubble has the observed dimensions, that it expands subsonically and that it is not buoyant. This situation is similar to what we refer to as a weakly or not overpressured regime. The great difference between our low power cases and a hot underdense bubble is the presence of the highly collimated jet that forms a thermal hot spot and is likely not effective in uplifting the cooling flow gas as proposed for example by Soker et al. (2002) to explain the presence of cool gas in the rims around the cavities. A collimated jet tends to go through the gas of the cluster displacing it aside and without uplifting it. Once the jet has terminated its active phase is rapidly destroyed (Reynolds et al. 2002) and the lobes can evolve like buoyant bubbles (see for example Churazov et al. 2001 or Quilis et al. 2001). An example similar to Perseus A is the Hydra A cluster containing the powerful FR I radio source $3 \mathrm{C} 218$. Given the properties of the X-Ray gas $\left(a \sim 26 \mathrm{kpc}, n_{0} \sim 0.06 \mathrm{~cm}^{-3}, T \sim 3.1 \mathrm{keV}\right.$, David et al. 2001) and a cocoon length of $\sim 49 \mathrm{kpc}$ we obtain an upper limit on the jet power $L_{\mathrm{j}} \sim 1.1 \times 10^{46} \mathrm{erg} \mathrm{s}^{-1}$. Since there is no indication that the gas surrounding the radio lobes is hotter than the ambient cluster gas this limit can be lowered at least by an order of magnitude. On the other hand it is possible to find some examples of radio sources inside galaxy clusters that do not show the presence of X-ray cavities. In our scheme these sources could correspond to radio lobes still in a strongly overpressured phase. For example the FR II radio source 3C 295 is found inside a cluster whose core is characterized by a radius $a \sim 17.8 \mathrm{kpc}$, a central density $n_{0} \sim 0.16 \mathrm{~cm}^{-3}$ and a temperature $T \sim 3.7 \mathrm{keV}$ (Allen et al. 2001). This cluster does not show any cavity in its X-ray emission. Given the longitudinal dimension of the radio source $\sim 17.6 \mathrm{kpc}$ we can estimate a lower limit of the jet power $L_{\mathrm{j}} \sim 7.1 \times 10^{45} \mathrm{erg} \mathrm{s}^{-1}$.

\section{Summary}

In this paper we performed numerical simulations of axisymmetric supersonic jets propagating in an isothermal background atmosphere. In agreement with the results presented by Reynolds et al. (2001), we find two distinct and subsequent regimes of interaction between the cocoon and the external medium. In the first phase of evolution, the overpressured cocoon drives a strong shock in the ambient medium, forming a thin, hot and compressed shell of shocked material, in the second phase the shock becomes very weak and the shell widens, decreasing its density and temperature. The resulting X-ray 
morphology in the two phases is different: in the strongly overpressured phase, we expect a shell of enhanced X-ray emission surrounding the radio emitting material, while, in the weak shock phase, we expect a deficit of X-ray emission coincident with the radio lobes. We have studied the dependence of the transition between these two phases on the physical jet parameters, by a wide coverage of the parameter space and by a comparison of the results of numerical simulations with analytical models. We find that the transition length between the two regimes depends essentially only on the jet power scaled over a value dependent on the properties of the ambient medium.

Acknowledgements. The authors acknowledge the italian MIUR for financial support, grant No. 2001-028773. The numerical calculations have been performed at CINECA in Bologna, Italy, thanks to the support of INAF.

\section{Appendix A}

As we have discussed, the jet replenishes the cocoon of matter and energy, therefore the cocoon expands compressing the ambient medium and if the expansion speed of the cocoon is highly supersonic, it will drive a strong shock in the external medium. The first attempts to give an analytic description of theses processes was done by Begelman \& Cioffi (1989) and Cioffi \& Blondin (1992). To describe the energy input by the jet, they write the following energy equation for the extended cocoon

$\frac{\mathrm{d} E_{\mathrm{c}}}{\mathrm{d} t}=L_{\mathrm{j}}$,

where $E_{\mathrm{c}}$ is the total energy in the extended cocoon and $L_{\mathrm{j}}$ is the jet power. Assuming a constant energy input by the jet and that all the energy is converted in thermal energy, we can then write the average (extended) cocoon pressure as

$P_{\mathrm{c}}=\frac{(\gamma-1) L_{\mathrm{j}} t}{V_{\mathrm{c}}}$

where $V_{\mathrm{c}}$ is the volume of the (cylindrical) cocoon equal to $\pi r_{\mathrm{e}}^{2} l_{\mathrm{c}}, r_{\mathrm{e}}$ is the extended cocoon radius and $l_{\mathrm{c}}$ the cocoon length assumed to increase as $v_{\mathrm{h}} t$, with $v_{\mathrm{h}}$ given by the onedimensional estimate $v_{\mathrm{h}}=\sqrt{v} v_{\mathrm{j}} /(1+\sqrt{v})$, where $v=\rho_{\mathrm{j}} / \rho_{\text {ext }}$. The lateral expansion of the extended cocoon can then be obtained by assuming a strong lateral shock, for which we can write

$\frac{\mathrm{d} r_{\mathrm{e}}}{\mathrm{d} t}=\sqrt{\frac{\gamma+1}{2} \frac{P_{\mathrm{c}}}{\rho_{\mathrm{ext}}}}$,

where $\rho_{\text {ext }}$ is the external density. Equation (A.3) can be integrated giving for the time behavior of $r_{\mathrm{e}}$ a dependence on $t^{1 / 2}$ and for $P_{\mathrm{c}}$ a dependence on $t^{-1}$. The behavior of the cocoon proper can then be obtained by writing its energy balance, that will differ from Eq. (A.1) by the term describing the work done at the contact discontinuity by the cocoon on the external medium and will have the form

$\frac{\mathrm{d} E_{\mathrm{ci}}}{\mathrm{d} t}=L_{\mathrm{j}}-P_{\mathrm{c}} \frac{\mathrm{d} V_{\mathrm{ci}}}{\mathrm{d} t}$, where $E_{\mathrm{ci}}$ is now the total energy of the cocoon proper and $V_{\mathrm{ci}}$ its volume. This equation can be written in an integral form, neglecting the initial value of the volume:

$V_{\mathrm{ci}}=\frac{1}{P_{\mathrm{c}}(t)^{1 / \gamma}}\left\{\frac{\gamma-1}{\gamma} \int_{0}^{t} L_{\mathrm{j}} P_{\mathrm{c}}^{(1-\gamma) / \gamma} \mathrm{d} t\right\}$.

Inserting in this equation the expression for the pressure derived above and assuming that the volume $V_{\mathrm{ci}}$ is proportional to $l_{\mathrm{c}} r_{\mathrm{i}}^{2}$ and the cocoon length behaves in the same way as that of the extended cocoon we can derive that the cocoon radius $r_{\mathrm{i}}$ behaves as $t^{1 / 2}$, in the same way as $r_{\mathrm{e}}$. In this situation we will then have a thin shell whose relative thickness stays constant. This description is well suited for strongly overpressured cocoons that drive strong shocks in the ambient medium, but the temporal dependence of pressure $\left(\propto t^{-1}\right)$ tells us that a cocoon cannot stay during all its evolution in a strongly overpressured regime. In addition there might be values of the parameters for which the cocoon is not strongly overpressured already at the beginning of its evolution. For describing these situations we have to extend Begelman \& Cioffi (1989) model. We need to reconsider the energy balance of the extended cocoon, observing that in addition to the energy input by the jet, the extended cocoon acquire also the thermal energy of the external material that enters the extended cocoon during its expansion. We can then write

$E_{\mathrm{c}}=L_{\mathrm{j}} t+\int_{V_{\mathrm{c}}} \frac{P_{\text {ext }}}{\gamma-1} \mathrm{~d} V$

where the second term on the right hand side represents the contribution described above and $P_{\text {ext }}$ is the pressure of the external medium. The equation for the pressure, instead of Eq. (A.2) becomes

$P_{\mathrm{c}}=P^{*}+\frac{(\gamma-1) L_{\mathrm{j}} t}{V_{\mathrm{c}}}$,

where

$P^{*}=\frac{1}{V_{\mathrm{c}}} \int_{V_{\mathrm{c}}} P_{\text {ext }} \mathrm{d} V$.

In the case of uniform external pressure, we have, of course, that $P^{*}=P_{\text {ext }}$. A second extension to the Begelman \& Cioffi (1989) model is done by considering a lateral shock of arbitrary strength and writing the expansion speed of the cocoon in a more general way as (see e.g. Landau \& Lifshitz 1959)

$\left(\frac{\mathrm{d} r_{\mathrm{e}}}{\mathrm{d} t}\right)^{2}=c_{\mathrm{se}}^{2}\left(\frac{\gamma-1}{2 \gamma}+\frac{\gamma+1}{2 \gamma} \frac{P_{\mathrm{c}}}{P_{\mathrm{ext}}}\right)$.

The system of Eqs. (A.6-A.8) can be solved analytically in the case of uniform external pressure assuming a cocoon length $l_{\mathrm{c}} \sim v^{1 / 2} v_{\mathrm{j}} t$ and $L_{\mathrm{j}}=\pi / 2 r_{\mathrm{j}}^{2} \rho_{\mathrm{j}} v_{\mathrm{j}}^{3}$, i.e. the kinetic jet power. This approximation is strictly valid for jets that are greatly supersonic with respect to their internal sound speed, so as to neglect the enthalpy flux term. Neglecting its initial value $\sim r_{\mathrm{j}}$ (jet radius), the cocoon radius then is given by

$$
\begin{aligned}
r_{\mathrm{e}}^{2} & =c_{\mathrm{se}}^{2} t^{2}+\sqrt{\gamma^{2}-1} r_{\mathrm{j}} v^{1 / 4} M c_{\mathrm{se}} t \\
& =r_{\mathrm{j}}^{2}\left(\frac{1}{v M^{2}} \frac{l_{\mathrm{c}}^{2}}{r_{\mathrm{j}}^{2}}+\frac{\sqrt{\gamma^{2}-1}}{v^{1 / 4}} \frac{l_{\mathrm{c}}}{r_{\mathrm{j}}}\right)
\end{aligned}
$$


and the cocoon pressure is

$$
\begin{aligned}
\frac{P_{\mathrm{c}}}{P_{\mathrm{ext}}} & =1+\frac{\gamma(\gamma-1)}{2}\left(\frac{1}{v^{1 / 2} M^{2}} \frac{c_{\mathrm{se}}^{2} t^{2}}{r_{\mathrm{j}}^{2}}+\frac{\sqrt{\gamma^{2}-1}}{v^{1 / 4} M} \frac{c_{\mathrm{se}} t}{r_{\mathrm{j}}}\right)^{-1} \\
& =1+\frac{\gamma(\gamma-1)}{2}\left(\frac{1}{v^{3 / 2} M^{4}} \frac{l_{\mathrm{c}}^{2}}{r_{\mathrm{j}}^{2}}+\frac{\sqrt{\gamma^{2}-1}}{v^{3 / 4} M^{2}} \frac{l_{\mathrm{c}}}{r_{\mathrm{j}}}\right)^{-1} .
\end{aligned}
$$

Substituting the solution of the pressure in the weakly overpressured regime in Eq. (A.5), we obtain that the internal radius begins to expand subsonically and tends asymptotically to a constant value.

\section{References}

Alexander, P. 2002, MNRAS, 335, 610

Allen, S. W., Taylor, G. B., Nulsen, P. E. J., et al. 2001, MNRAS, 324, 842

Begelman, M. C., \& Cioffi, D. F. 1989, ApJ, 345, L21

Blanton, E. L., Sarazin, C. L., McNamara, B. R., \& Wise, M. W. 2001, ApJ, 558, L15

Böhringer, H., Voges, W., Fabian, A. C., Edge, A. C., \& Neumann, D. M. 1993, MNRAS, 264, L25

Böhringer, H., Nulsen, P. E. J., Braun, R., \& Fabian, A. C. 1995, MNRAS, 274, L67

Brighenti, F., \& Mathews, W. 2002, ApJ, 574, L11

Carilli, C. L., Perley, R. A., \& Harris, D. E. 1994, MNRAS, 270, 173

Churazov, E., Forman, W., Jones, C., \& Böhringer, H. 2000, A\&A, 356, 788

Churazov, E., Brüggen, M., Kaiser, C. R., Böhringer, H., \& Forman, W. 2001, ApJ, 554, 261
Cioffi, D. F., \& Blondin, J. M. 1992, ApJ, 392, 458

Clarke, D. A., Harris, D. E., \& Carilli 1997, MNRAS, 284, 981

David, L. P., Nulsen, P. E. J., McNamara, B. R., et al. 2001, ApJ, 557, 546

Fabian, A. C., Sanders, J. S., Ettori, S., et al. 2000, MNRAS, 318, L65

Fabian, A. C., Celotti, A., Blundell, K. M., Kassim, N. E., \& Perley, R. A. 2002, MNRAS, 331, 369

Finoguenov, A., \& Jones, C. 2001, ApJ, 547, L107

Heinz, S., Reynolds, C. S., \& Begelman, M. C. 1998, ApJ, 501, 126

Heinz, S., Choi, Y., Reynolds, C. S., \& Begelman, M. C. 2002, ApJ, 569, L79

Krause, M. 2003, A\&A, 398, 113

Landau, L. D., \& Lifshitz, E. 1959, Fluid Mechanics (London: Pergamon)

Massaglia, S., Bodo, G., \& Ferrari, A. 1996, A\&A, 307, 997

McNamara, B. R., Wise, M., Nulsen, P. E. J., et al. 2000, ApJ, 534, L135

Nulsen, P. E. J., David, L. P., McNamara, B. R., et al. 2002, ApJ, 568, 163

Owen, F. N., \& Eilek, J. A. 1998, ApJ, 493, 73

Quilis, V., Bower, R. G., \& Balogh, M. 2001, MNRAS, 328, 1091

Reynolds, C. S., Heinz, S., \& Begelman, M. C. 2001, ApJ, 549, L179

Reynolds, C. S., Heinz, S., \& Begelman, M. C. 2002, MNRAS, 332, 271

Rizza, E., Loken, C., Bliton, M., Roettiger, K., \& Burns, J. O. 2000, AJ, 119, 21

Schmidt, R. W., Fabian, A. C., \& Sanders, J. S. 2002, MNRAS, 337 , 71

Smith, D. A., Wilson, A. S., Andrew, S., et al. 2002, ApJ, 565, 195

Soker, N., Blanton, E. L., \& Sarazin, C. L. 2002, ApJ, 573, 533

Woodward, P. R., \& Colella, P. 1984, J. Comp. Phys., 54, 174 